\title{
A constrained optimization problem under uncertainty
}

\author{
Keivan Shariatmadar, Erik Quaeghebeur \& Gert de Cooman \\ Ghent University, SYSTeMS Research Group \\ \{Keivan. Shariatmadar, Erik. Quaeghebeur, Gert.deCooman\}@UGent.be
}

\begin{abstract}
Consider the following optimization problem: maximize a bounded real-valued function $f$ - defined on a set $\mathscr{X}$ - over all $x$ in $\mathscr{X}$ that satisfy the constraint $x R Y$, where $Y$ is a random variable taking values in a set $\mathscr{Y}$ and $R$ is a relation on $\mathscr{X} \times \mathscr{Y}$. The aim of our active research is to reduce this problem to a (constrained) optimization problem from which the uncertainties present in the description of the constraint are eliminated.

We investigate what results can be obtained for different types of uncertainty models for the random variable $Y-$ linear previsions, vacuous previsions, possibility distributions, p-boxes, etc. [see, e.g., 1, 2, 4] - and for two different optimality criteria - maximinity and maximality [see, e.g., 3]. We work with general $\mathscr{X}, \mathscr{Y}$, and $R$ for the combinations of uncertainty model and optimality criterion that allow it and restrict our attention to more concrete situations otherwise.

In our poster, we will present the problem statement, give illustrated solutions for the most interesting cases we have investigated, as well as discuss the strengths and weaknesses of our approach. In the remainder of this abstract, we sketch this approach and give the solutions of two of the cases we have already fully investigated.

The first thing we do is reformulate the optimization problem as a decision problem. To wit, with every $x$ in $\mathscr{X}$ we associate a utility function $G_{x}$ on $\mathscr{Y}$ that gives the constant value $f(x)$ for $y$ in $\mathscr{Y}$ such that $x R y$ and that gives a penalty-value $L$ otherwise (i.e., when $x \not R y)$. As the name suggest, it penalizes the fact that $x$ such that $x \not R y$ are considered; we therefore choose $L<\inf f$. The uncertainty model associated to $Y$ is formulated as a coherent lower prevision $\underline{P}$ (and its conjugate upper prevision $\bar{P}$ ) for a sufficiently rich set of gambles on $\mathscr{Y}$ [see, e.g., 4, for terminology]. Using the maximinity criterion, the optimal values for $\mathscr{X}$ are those that maximize $\underline{P}\left(G_{x}\right)$. Using the more conservative maximality criterion, the optimal values for $\mathscr{X}$ are those for which $\min _{z \in \mathscr{X}} \bar{P}\left(G_{x}-G_{z}\right) \geq 0$. Both finding the prevision on the sufficiently rich set of gambles $\left\{G_{x}: x \in \mathscr{X}\right\}$ or $\left\{G_{x}-G_{z}: x, z \in \mathscr{X}\right\}$ and checking the optimality criteria are, in general, nontrivial steps.
\end{abstract}

For the general case, when $Y$ is described by a linear prevision $P$, we find that under both maximinity and maximality the optimal $x$ are those that maximize the function on $\mathscr{X}$ that takes the value $(f(x)-L) P(\{y \in \mathscr{Y}: x R y\})$ in $x$.

For the concrete case $\mathscr{X}=\mathscr{Y}:=\mathbb{R}$ and $R:=\leq$, and when $Y$ is described by a triangular possibility distribution with basis $[a, b] \subset \mathbb{R}$ and top $c \in(a, b)$, we find that under maximinity the optimal $x$ are those that maximize the function on $(-\infty, c]$ that takes the value $f(x)$ for $x \leq a$ and $\frac{x-a}{c-a} L+\frac{c-x}{c-a} f(x)$ for $x$ in $(a, c]$.

Keywords. Constrained optimization, maximinity, maximality, linear prevision, vacuous prevision, possibility distribution, p-box.

\section{References}

[1] Didier Dubois and Henri Prade. Théorie des possibilités. Masson, 2nd edition, 1988.

[2] Scott Ferson, Vladik Kreinovich, Lev Ginzburg, Davis S. Myers, and Kari Sentz. Constructing probability boxes and Dempster-Shafer structures. Technical Report SAND2002-4015, Sandia National Laboratories, 2002.

[3] Matthias C. M. Troffaes. Decision making under uncertainty using imprecise probabilities. International Journal of Approximate Reasoning, 45(1):17-29, 5 2007. doi:10.1016/j.ijar.2006.06.001.

[4] Peter Walley. Statistical Reasoning with Imprecise Probabilities. Chapman and Hall, London, 1991. 